\title{
Bayesian confirmation, connexivism and an unkindness of ravens*
}

\author{
Elisángela Ramírez-Cámara \\ Institute for Philosophical Research, UNAM \\ Mexico City, Mexico \\ eliramirezc@gmail.com
}

\begin{abstract}
Bayesian confirmation theories (BCTs) might be the best standing theories of confirmation to date, but they are certainly not paradox-free. Here I recognize that BCTs' appeal mainly comes from the fact that they capture some of our intuitions about confirmation better than those theories that came before them and that the superiority of BCTs is sufficiently justified by those advantages. Instead, I will focus on Sylvan and Nola's claim that it is desirable that our best theory of confirmation be as paradox-free as possible. For this reason, I will show that, as they respond to different interests, the project of the BCTs is not incompatible with Sylvan and Nola's project of a paradox-free confirmation logic. In fact, it will turn out that, provided we are ready to embrace some degree of non-classicality, both projects complement each other nicely.
\end{abstract}

\section{Introduction}

A somewhat overlooked application for relevant (and connexive) logics is offered in Sylvan and Nola's [41]. There, they show that replacing classical logic with one of these logics results in a paradox-free version of both instantial confirmation and hypothetico-deductivism. A salient bit of this proposal is that they tend to frame non-classical qualitative confirmation theories as a suitable alternative to Bayesian theories of confirmation. In this paper, I will argue that there is no need to choose one approach over the other.

*This research was supported by the PAPIIT project IA401117 "Aspectos filosóficos de las lógicas contraclásicas". also received financial support through Conacyt's National and Mixed Grants Program. The latter allowed me to finish this paper while visiting the University of Connecticut's Philosophy Department. I thank Manuel Tapia-Navarro, Claudia TanúsPimentel, María Martínez-Ordaz, Moisés Macías-Bustos, Alejandro Vázquez del Mercado and Thomas Ferguson for lending an ear when I needed it most, as well as providing valuable discussion. I have also benefited from the comments of two anonymous referees. I'm very grateful to Luis Estrada-González, especially for his enduring patience, his kind words of encouragement and for never failing to share a laugh with me, even during the toughest of times. 
On the one hand, Bayesian confirmation theories (BCTs) are not meant to provide explicit claims about logic, and their paradox-handling ability is actually a desirable byproduct of their main advantage: a finer grained notion of confirmation that allows, for instance, the expression of comparative claims. On the other hand, Sylvan and Nola's claim is explicitly about choosing the right logic for a specific purpose. Thus, there seems to be no incompatibility and no immediate reason to choose one over the other. Instead, this discussion seems to be more about shedding light on a single issue from different angles. If this is so, then the possibility of keeping both perspectives should be worth exploring.

After some preliminaries on both confirmation theories (Sect. 2) and the confirmation paradoxes (Sect. 3), both the Bayesian and non-classical (qualitative) strategies for paradox resolution are outlined in Sect. 4 . Then, Section 5 shows that even if BCTs have made great advances in dealing with the paradoxes better than their qualitative cousins, some issues remain. The upshot is that solving those issues by combining the Bayesian approach with Sylvan and Nola's non-classical solution has some benefits of its own, like the recovery of the unrestricted Nicod Condition. This section ends with some remarks on combining both strategies. Finally, some conclusions are offered.

\section{An incomplete map of confirmation theory}

Confirmation is an essential concept within philosophy of science. Due to the notion that one of the main tools of philosophy of science is logic (see, for example, [34], esp. chapter 3), we tend to believe that the only correct analysis of our intuitive concept is a logical one. Specifically, due to the nature of the intuitive concept and its relationship to topics such as induction and the testability of hypotheses, confirmation is closely related to logical entailment. However, we seem to lack a truly comprehensive analysis of confirmation. In this section, I will outline three families of problems that have been faced in the search for an adequate theory of confirmation. They can be summarized as follows:

1. What is the actual relationship between confirmation and entailment?

2. Should one prefer truth-functionality over probability?

3. What is the best solution to the paradoxes of confirmation?

In the remainder of this section, each of these questions and their usual answers will be briefly developed.

\subsection{Two ways of relating entailment and confirmation}

The first attempt to develop a theory of confirmation has at its core what is now known as 'instantial confirmation' $[12,13]$. The intuitive idea behind 
instantial confirmation is that a given piece of evidence e, along with suitable background information $\mathrm{k}$, confirms $\mathrm{H}$ iff either e and $\mathrm{k}$ entail $\mathrm{H}$, or e and $\mathrm{k}$ entail a statement $\mathrm{s}$ which in turn entails $\mathrm{H}$. The paradigmatic example of instantial confirmation is that of the ornithologist who wishes to find out whether all birds of a certain species are of the same color. Instantial confirmation satisfies at least the following five conditions (taken practically untouched from [36]):

Nicod condition (NC) A positive instance of a given hypothesis confirms it.

Entailment condition (EC) A piece of evidence e (along with some suitable background information) directly confirms $\mathrm{H}$ iff e $\wedge \mathrm{k} \Vdash \mathrm{H}$.

Consequence condition (CC) A piece of evidence e (along with some suitable background information) confirms $\mathrm{H}$ iff e $\wedge \mathrm{k} \Vdash \mathrm{s}$ and $\mathrm{s} \Vdash \mathrm{H}$. This condition entails the Special Consequence Condition (SCC): if e confirms $\mathrm{H}$, then e confirms any consequence of $\mathrm{H}$.

Equivalence condition (Eq) Whatever confirms a given hypothesis will confirm any logically equivalent statement of that hypothesis.

Classicality (CL) The correct language of a confirmation theory is that of (first-order) classical logic. ${ }^{1}$

Those five principles are enough to give rise to (al least) the infamous Raven Paradox.

However, independently from the paradoxes, instantial confirmation also lost its appeal because it cannot be enriched with

Converse Consequence Condition (CCC) If e confirms H, then e also confirms any $\mathrm{H}^{\prime}$ such that $\mathrm{H}^{\prime} \Vdash \mathrm{H}$.

without the unwelcome consequence that any statement can therefore confirm any other. However, (CCC) tells us something about the predictive power of hypotheses that is hard to give up.

Consequently, as an alternative to instantial confirmation, Hypotheticodeductive confirmation (HD-confirmation) is fully based on the idea that hypotheses are confirmed by their predictions. In a nutshell, HD-confirmation arises from the substitution of (EC) with an "actual" entailment condition, namely

Prediction condition (PC) e confirms $\mathrm{H}$ iff $\mathrm{H}$ (along with suitable background knowledge $\mathrm{k}$ ) entails e; that is, iff (1) $H \wedge k$ is consistent, (2) $H \wedge k \Vdash e$ and $(3) k \nVdash e$.

\footnotetext{
${ }^{1}$ This one is seldom made explicit, as it is typically taken as a given that classical logic must be the one operating in these contexts. However, as the proposal consists in contesting the only assumption that is taken as a given, it seems adequate to include it among the others.
} 
Thus, based on the way each proposal relates confirmation and entailment, instantial confirmation has a logical structure akin to that of universal generalization, HD-confirmation has a structure that looks very much like universal instantiation.

Typically, HD-confirmation is lauded as an alternative to instantial confirmation. But it is important to note, from above, that the core intuitions of both proposals are not only incompatible, but also fundamentally different. This could mean that they capture irreducible aspects of our intuitive notion of confirmation. So, while there might be compelling reasons to choose HDconfirmation over instantial confirmation, not everyone endorses this course of action. For example, Okasha [19] argues that while confirmation theories tend to talk about generic evidence confirming theories and hypotheses, there is a legitimate distinction to be traced between observational and experimental evidence in both traditional and Bayesian theories of confirmation. More precisely, it seems that purely observational reports take the shape $F a \wedge G a^{2}$, while experimental reports are those in which $F a$ is brought about beforehand, so that the final report is better construed as $F a \supset G a$ and is clearly entailed by the generalization.

Sylvan and Nola are also reluctant to do away with instantial confirmation. Their reasons are, however, independent from Okasha's. This is because they focus on the paradoxes of confirmation as grounds for the rejection of any theory of confirmation. In terms of being paradox free, we will see that HDconfirmation hardly fares better than instantial confirmation. The discussion of the next family of problems hinges on this apparently innocent remark, because Bayesian confirmation theories (BCTs) are often touted as our best shot at solving - or rather, explaining away - most paradoxes of confirmation. Thus, as the following brief overview will show, BCTs are pushed as an alternative to (qualitative) HD-confirmation and instantial confirmation theories.

\subsection{The unsurprising popularity of BCTs}

BCTs have risen into prominence as "[...] the leading account of induction and confirmation in the philosophy of science literature" [18], mainly because, unlike their antecessors, they provide us with quantitative analyses of confirmation. The flexibility of the resulting analysis comes into a stark contrast with the rigidity of qualitative analyses, in which confirmation tends to be an all-ornothing matter. The basis of BCTs is that support comes in degrees which are construed as degrees of belief in a given hypothesis (cf. [37]). The dynamics of the degrees of belief are then best understood as a probability calculus, where specific changes are obtained through Bayesian updating on a given probability distribution. This way of understanding confirmation is appealing because it lets us talk about things like having a piece of evidence confirm an hypothesis more than another.

Even if BCTs seem to recover the core of their qualitative rivals - see Crupi

\footnotetext{
${ }^{2}$ Which is not implied by the generalization, as it at most implies $\mathrm{Fa} \supset \mathrm{Ga}$.
} 
[4], and contrast probabilistic confirmation as firmness and probabilistic relevance confirmation - probabilistic relevance is often strongly preferred to firmness. I take this as telling of the following: (1) That we expect Bayesian confirmation to behave more like HD-confirmation than instantial confirmation; and (2) that there is a parallel to be made between (deductive) logical validity and a very general definition of Bayesian confirmation.

Bayesian confirmation, like material implication $(A \supset B)$ and logical consequence $(A \vdash B)$, is often thought of as a relation that preserves something between a pair of statements $\mathrm{A}$ and $\mathrm{B}$. The truth-functional definition of material implication we are familiar with tells us that implication is false every time it takes us from a true antecedent to a false consequent. Likewise, logical consequence can be thought of as a truth preserving relation, with invalid arguments being those which take us from a true premise set to conclusions that are not. Finally, Bayesian refutation happens when the posterior conditional probability of the hypotheses on the evidence (B) is lower than the initial probability of the hypotheses alone (A). So, it seems quite uncontroversial to say, as I did in the preceding section, that what all of these relations have in common is that they are about preserving something.

However, it is important to notice that material implication and Tarskian logical consequence can be seen strictly as avoiding the loss of truth, as they are silent about vacuous truths, namely, those which go from a false antecedent or premise set to a true consequent or conclusion (set). In contrast, confirmation is not only about the preservation of certainty, but also about the effect that the likelihood of some statements has on the likelihood of the hypotheses under consideration. This means that we have to rule out the analog phenomenon that could be described as "vacuous confirmation". This is achieved when we restrict our candidates for confirmation only to those which have an adequate prior degree of certainty. Regardless, even considering this restriction, this behaviour is similar enough for us to assume that the confirmation relation is closely related to the material conditional, so that a Bayesian framework is a sort of probabilistic (classical) logic.

Thus, we can understand the basis of the confirmation relation in the following way:

Bayesian confirmation $\mathrm{B}$ confirms $\mathrm{A}$ just in case that, if $\mathrm{A}$ has a positive probability, then B has a (positive) probability such that it won't decrease A's upon conditioning.

Or, in more familiar terms: B confirms A just in case conditioning A on B does not decrease the original certainty of A. Or, even better: B confirms A just in case that if A is somewhat likely, then B is not too unlikely.

So construed, it becomes tempting to simply treat the underlying logic as classical: with little to no effort, BCTs become a reasonable framework for probabilistic reasoning, as it allows to model degrees of certainty, or credences, instead of absolute certainty or uncertainty while allowing the preservation of 
all the alleged virtues of classical logic. ${ }^{3}$ In other words, it is safe to assume that (CL) underlies BCTs, as well as (NC) and (PC). ${ }^{4}$ Furthermore, this way of construing Bayesian confirmation also goes nicely with a concern that has risen at several points of the discussion, namely, that of the status of (EC) and (CCC). While it is unclear whether BCTs explicitly recover either condition, they do seem to uphold the idea that confirmation (and induction) are the converse of deduction. So, to summarize, an important part of the appeal of $\mathrm{BCTs}$ comes from the fact that they uphold the very same conditions their rivals do.

\section{The paradoxes of confirmation: a brief re- fresher}

Thus far, what seems to be especially appealing about BCTs is that they offer a more fine-grained analysis of our notion of confirmation while preserving the intuitive conditions which underlie both instantial and HD-confirmation. But also of particular interest is the fact that BCTs are also praised for "[their] epicycling elegance [...] in the face of paradoxes" [41, p. 14]. For this reason, before going fully into the discussion of Sylvan and Nola's work and its relationship with BCTs, the remainder of this one will be dedicated to a brief overview of the paradoxes that one could expect to be solved by any confirmation theory, Bayesian or otherwise.

\subsection{The (infamous) Raven paradox}

The Raven paradox ([12, 13] [14]) first arose in the context of instantial confirmation, and because of this, involves the conditions associated with this kind of confirmation. Specifically, the paradox begins with the observation that something is an $\mathrm{F}$ and a $\mathrm{G}(\mathrm{Fa} \wedge \mathrm{Ga})$, which by $(\mathrm{NC})$ confirms the generalization that all Fs are Gs. Then, by (EQ) and (CL) we have that, as "All Fs are Gs" is logically equivalent to "Every not-G is a not-F", then the observation confirms both hypotheses equally. That the reasoning involved in this argument leads us to a "paradoxical" conclusion can be better appreciated by way of the famous example:

The assertion that "All ravens are black", is an A-proposition, better known as universal generalization in first-order classical logical. Because we are taking (CL) to hold, this statement is logically equivalent to its contrapositive "All nonblack things are non-ravens". According to the usual story, the latter statement is confirmed by practically anything but ravens. Furthermore, by (EQ) we have

\footnotetext{
${ }^{3}$ It is important to notice that in this context, logic is no longer about truth and falsity, as we are dealing with belief states rather than with correspondence with the world or something similar. Although truth and certainty are not equivalent, for now we can say that, ideally, the things a given rational agent is certain about are those which are in fact true.

${ }^{4}(\mathrm{PC})$ holds as long as we prefer relevance to firmness.
} 
that whatever confirms the original assertion also confirms any statement which is logically equivalent to it. Thus, we get the following

Paradoxical conclusion (Par) Black ravens confirm "All ravens are black" as much as things which are neither ravens nor black (such as pink flamingoes) do.

We call (PAR) paradoxical because we think that our original generalization has nothing to do with things that are neither black nor ravens. That is, we seem to share the intuition that only such things as ravens are in some sense relevant for the evaluation of whether all ravens are black.

One of the successes of HD-theories is that they provide a sufficient criterion for blocking the paradox in the shape of 'auxiliary hypotheses' or 'background information'. In the particular case of "All ravens are black", the sighting of a black object will confirm the generalization only under the assumption that it was taken from among the ravens. Likewise, a non-raven will confirm the very same generalization, only relative to the assumption that the object was taken from the set of the non-black things. However, we already saw that this does away with another intuition we would like to preserve. Under this view, evidential statements can no longer have the shape $R a \wedge B a$. If the above is reconstructed as a sort of conditional proof, then neither can we obtain statements of this shape using this method, nor are they counted among the (logical) predictions of the hypothesis or consequences of the evidential statements.

\subsection{Irrelevant conjunctions}

But, even if HD-confirmation can satisfactorily block the Raven paradox (at some cost), it is not able to remain paradox-free. The most notorious of the paradoxes that afflict HD-confirmation is the irrelevant conjunct or tacking paradox. This one arises because from (CL) it follows that the consequence relation is assumed to be monotonic, and because of (SCC). Thus, if for e to confirm $\mathrm{h}$ (relative to some background information $\mathrm{k}$ ) means that $h \wedge k \Vdash e$ should hold, then by monotonicity of entailment, $h \wedge j \wedge k \Vdash e$ holds. This means that, if e confirms $\mathrm{h}$, then it also confirms $h \wedge j$ and, by an application of Simplification, also confirms $\mathrm{j}$, for any $\mathrm{j}$ whatsoever. ${ }^{5}$ The move is highly suspicious as there are actually no restrictions on what $\mathrm{j}$ should be, as long as validity is preserved. This problem is similar to the trivialization of instantial confirmation through the addition of $(\mathrm{CCC})$.

\section{3 "Gruesome" paradoxes}

Additionally, HD-confirmation is unable to get rid of a final family of paradoxes, namely, those introduced by Goodman (anticipated by Russell) and their kin. The extensional variant of the grue paradox is logically quite similar to the

\footnotetext{
${ }^{5}$ In fact, when restricting oneself to validity preservation, it also follows that e confirms the background information $\mathrm{k}$.
}

Australasian Journal of Logic (15:2) 2018 Article no. 3.10 
Raven paradox, as, by (NC) every observation of green emeralds up to a time $\mathrm{t}$ confirms the hypothesis "All non-blue emeralds are examined before t". Unfortunately, by (CL) and (EQ) we have that those same observations confirm the logically equivalent hypothesis "All emeralds not examined before t are blue". The definitional variant of the grue paradox, along with the paradoxes are shown by Sylvan and Nola to involve direct applications of, most importantly, Disjunctive Syllogism and Addition. None of the requirements for either the extensional or the definitional paradoxes falls outside the scope of what we are taking to be rock-solid conditions for a satisfactory theory of confirmation. Thus, here as in all other cases of paradox, if there is an explanation as to what is going wrong, it must be elsewhere. Or else there might be none, and grue-like paradoxes must be taken for what they are: unpleasant consequences of otherwise acceptable theories of confirmation.

\section{Two (apparently) competing accounts of para- dox resolution}

The overview of the paradoxes of confirmation just developed was prompted by the statement that one of the advantages of BCTs over their qualitative rivals is that the former are the best behaved of the three in the face of paradox. It is precisely at this point that the importance of Sylvan and Nola's work shines through. For while it is true that BCTs are able to handle the paradoxes of confirmation better than their rivals, it will turn out that BCTs only count as the best alternative for a comprehensive theory modulo the assumption that they are better suited to restrict those principles other than (CL) at play. Sylvan and Nola's key insight is precisely that there seem to be no good reasons to preserve (CL); on the contrary, there is actually much to be gained with its rejection.

\subsection{The Bayesian strategy}

This way of framing things is not exactly new. An anonymous referee pointed me towards [8]. There, Branden Fitelson argues for a reconstruction of Goodman's argument against instantial confirmation as involving a premise which contains at least a suitable formulation of instantial confirmation theory and another which is a bridge principle that connects confirmation (a logical notion) with evidential support (an epistemic notion). If this is so, then the advocates of instantial confirmation can either modify an aspect of the theory or reject the bridge principle.

Fitelson, as many, believes that offering a Harman-like response is the way to go. ${ }^{6}$ However, he also doubts that instantial confirmation theorists will be

\footnotetext{
${ }^{6}$ It's important to notice that while both Fitelson and the anonymous referee who pointed me to this paper focus on grue-like paradoxes, providing an alternative theory that does not require a bridge principle would not only give us grounds to reject these, but also nearly every other paradox discussed here.
} 
able to offer a convincing response of this kind. In contrast, he does think that Bayesians will be able to provide such a response. To what does the response amount? It amounts to the rejection of the Total Evidence Requirement (RTE). Fitelson endorses the full rejection of (RTE) where others are more modest and have only tried to reformulate it. Nonetheless, even he recognizes the difficulties involved in offering a suitable alternative, and thus focuses on what he calls "Goodman's Quantitative Argument".

This shift on perspective is important, because allows me to draw a general picture of the most developed Bayesian strategy for solving both the Raven Paradox and those involving grue-like predicates. When discussing how Bayesians could fight back against the claim that green emeralds confirm the greeness and the grueness of emeralds equally, he says that the best response available, that emeralds being green is a priori more probable than their being grue, is not the most satisfying answer. It is also not that different from Goodman's own proposal, that "green" is a more entrenched predicate than "grue".

This discussion squares quite nicely with Susanna Rinard's recent resolution to the Raven paradox [26] based on the naturalness of the predicates involved. Of course, there are plenty of interesting technicalities involved in her solution that I cannot discuss here. What I want to focus on is that Rinard's discussion, as was the case with Fitelson's falls back to grounding the preference of some predicates over others on features such as "naturalness" or "entrenchment".

While it is true that in one case the preference keeps the same piece of evidence from confirming two incompatible hypotheses, in the other the preference works to keep certain instances from confirming (or disconfirming) an hypothesis. However, it is not difficult to notice a couple of things. The first one is that even if "naturalness" and "entrenchment" are not exactly the same thing, they are meant to provide epistemic grounds for preferring certain sorts of predicates over others. They also ultimately fulfill the same role: they restrict (NC) so we are able to account for cases in which not every instance should count as confirmatory (Raven Paradox), and for extreme cases in which no instance should count as confirmatory (grue).

\subsection{The non-classical strategy}

Another reason why the change of perspective is important is that if I have reconstructed it along the right lines, then it sort of echoes other contexts in which proposals for non-classical solutions have also arisen. The naïve set theory and semantic paradoxes arise when the naïve non-logical principles (Comprehension, transparent truth) involved are inconsistent with the logical ones ((CL) and (EQ) or some suitable variants thereof). Each of these kinds of paradox has its own 'canonical' solution, which involves restricting the naïve principles, while leaving the logical ones intact.

Non-classical solutions arise from the motivation of leaving the naïve principles intact. This is so because, aside from the fact that they turn out to be inconsistent with the rest of the theory's commitments, most naïve principles tend to come across as quite harmless and intuitive. Granted, neither naïve set 
theory nor truth theory have to deal with a bridge principle in the way confirmation theory does. As a result, it makes more sense for those discussions to be construed as a matter of choosing which of the available principles of the theory would be less costly to reject. Thus, my only contention is that, pending the development of a radically different confirmation theory that makes no use of bridge principles, we can frame the current canonical solutions to the confirmation paradoxes in the same fashion as the naïve set theory and semantical paradoxes.

With this framing of the discussion, it is now evident how Sylvan and Nola's proposal comes into play. Contrary to what advocates of the canonical solution think, they simply find the rejection of (CL) as less costly than that of (NC). Their proposal, as expected, consists in making what they refer to as a "relevant switch", namely, adopting a relevant logic as the formal language that underlies a given confirmation theory (following Sylvan's own proposal in Ultralogic as Universal?(reprinted as an appendix in [32])). ${ }^{7}$ Their strategy consists entirely in showing how every single paradox involves a principle eschewed by relevantists, under the assumption that they are independently suspicious. Thus, the paradoxes of confirmation become yet another instance of a successful application of a relevant logic as a substitute of classical logic. However, it is worth noting that Sylvan and Nola's project is not without wrinkles, as one of the characteristic paradoxes of HD-confirmation - that of irrelevant conjunctionsis not solved by the adoption of a (narrowly) relevant logic, but requires the apparatus of connexive logic instead.

In what follows, I will briefly show how Sylvan and Nola's proposal effectively blocks each of the paradoxes discussed.

\subsubsection{The unkindness of ravens}

We are already familiar with what happens here. A pesky classically valid equivalence, Contraposition forces us to admit, much to our dismay, that every single object that is neither black nor a raven has as much to do with the confirmation of an hypothesis about the color of ravens as much as black ravens do. Sylvan and Nola examine to two different ways to break the logical equivalence between both conditionals. On their first attempt, they go after Contraposition. Unfortunately, Contraposition turns out to be too hard (but not impossible) to give up for two reasons. The first one is that a relevant implication weak enough to invalidate Contraposition is likely too weak to be useful. The other reason is that, although Contraposition has been tested under the weight of alleged counterexamples, there doesn't seem to be a sufficiently compelling case for its

\footnotetext{
${ }^{7} \mathrm{An}$ anonymous referee asked about the possibility of going substructural in order to solve these paradoxes. Relevant logics are a kind of relevant logics, as Weakening fails for intensional premise combination (see [22], footnote 4). Priest's footnote also explains the difference between relevant and non-monotonic logics proper, which as the referee notes, are not that popular for paradox resolution. Non-contractive logics such as linear logic are relevant logics that restrict both Contraction and Weakening. However, I see no need to go that weak when solving the confirmation paradoxes through non-classical logical means.
} 
rejection. ${ }^{8}$

Everything is not lost, however, because one can argue, as Sylvan and Nola do, that hypotheses such as "All ravens are black" are enthymematic, because they usually supress some sort of background information or ceteribus paribus clauses. Once this becomes evident, the realization is that the suspicious rule involved in this paradox is not Contraposition but Antilogism, which is relevantly invalid for extensional conjunctions. The failure of Antilogism lets us see clearly why the HD-confirmation to the solution of the Raven paradox works. As was seen the key to this solution of the paradox is the background information. As those who endorse HD-confirmation accept (CL) without question, they depend on the background information to contain the conditions under which each of the logically equivalent hypotheses is confirmed.

This is more problematic than it seems, as this can be said to demand too much from background information. For how should one go about formulating background information in a way that is sufficient to avoid paradox and not ad hoc? Take, once again the case of the Raven paradox. We are assuming that the hypothesis is correctly formalized as $(X \wedge R) \supset B$, where $\mathrm{X}$ is the background information and $\mathrm{R}$ is shorthand for the universal generalization. Then, through Antilogism, we get $(X \wedge \neg B) \supset \neg R$. Thus, in order for the HD-confirmation solution to go through, $\mathrm{X}$ must contain all the conditions that state which evidential reports confirm which hypotheses. Contrast this strategy with the rejection of Antilogism, which simply amounts to recognizing that, given some specific background information $\mathrm{X}, R \supset B$ and $\neg B \supset \neg R$ are in general not interchangeable, presumably because, following our intuitions, they don't say the same thing.

Interestingly, stronger relevant logics such as $\mathrm{R}$ let us recover some of the intuitions of the HD-confirmation solution. If one is able to define background theory as required, then one may make use of the valid related principle which states that $(X \circ R) \rightarrow B$ is equivalent to $(X \circ \neg B) \rightarrow \neg R$. This is not the same as using Antilogism, for the connective involved here - fusion - is an intensional, rather than extensional conjunction. ${ }^{9}$ For example, Simplification is invalid, which means that it is impossible to isolate an hypothesis from background theory, vindicating Quine-Duhem underdetermination and going against the core of HD-confirmation. ${ }^{10}$

\footnotetext{
${ }^{8}$ I understand 'compelling' in the same sense as Sylvan and Nola do: while no one doubts that all the technicalities are in place, the case is not compelling because restricting Contraposition seems to be very unlike what speakers of a natural language do.

${ }^{9}$ See [25] for an examination of the relevant logic argument against Antilogism and the distinction between this rule and the one involving fusion. I call fusion a conjunction, because while I don't have an account of how to define the connectives myself, I think the correct account is one that classifies fusions together with classical (extensional), additive and multiplicative conjunctions.

${ }^{10}$ Namely, that it is always possible to determine that a hypothesis along with some background information entails the evidence, which is not entailed by the background information alone.

An anonymous referee worries that in requiring an intensional bind between the hypothesis and the background information I'm going against the standard position regarding the structure of scientific theories. I think this issue can safely be avoided if one distinguishes the
} 


\subsection{Grue, blite and the like}

For the this family of paradoxes, Sylvan and Nola diagnose the problem with both the extensional and definitional variants of the grue paradox to be the suspicious application of Antilogism and Disjunctive Syllogism, respectively. ${ }^{11}$ As was stated above, the extensional version of the paradox involves the hypotheses "All non-blue emeralds are examined before t" and "All emeralds examined after $\mathrm{t}$ are blue" involve a relevantly-invalid application of Antilogism, as they are best formalized as going from $(E a \wedge \neg B a) \supset T a$ to $(E a \wedge \neg T a) \supset B a$. This places us in a situation which is analog to the one of the Ravens Paradox, and thus, is solved by the same token.

On the other hand, the definitional version of the paradox, along with its cousins such as blite and emerose, require a different strategy, but one that is equally invalid in the context of relevant logic. The solution for the definitional version of grue - recovered from Bass [1] by Sylvan and Nola - goes as follows: The observation in t1 of a green emerald prompts the acceptance of $G a \wedge T 1 a$. Then, by Addition, we can capture the reasoning behind the inference from the fact that an emerald is green when examined at t1 to the fact that its grue, namely, green when examined at t1 or blue when examined aferwards $((G a \wedge T 1 a) \supset((G a \wedge T 1 a) \vee(B a \wedge \neg T 1 a)))$. Then, given that there are emeralds that are not examined (and thus not found to be green), it seems reasonable to admit that if there is an emerald not examined $(\neg(G b \wedge T 1 b))$ and the disjunction holds for all emeralds $((G b \wedge T 1 b) \vee(B b \wedge \neg T 1 b))$; that emerald can be predicted to be blue $((B b \wedge \neg T 1 b))$. The other paradoxes in this family (blite and emerose) will not be revisited here. It suffices to say that, along with the definitional version of grue, the reasoning behind each paradox crucially depends on an application of Disjunctive Syllogism, which is not valid in the context of relevant logic.

\subsection{On to uncharted connexive territory}

The paradox of the irrelevant conjuncts is especially demanding because no relevant logic can solve it by logical means alone. The key rule here is Simplification, which is preserved by even the weakest relevant logics. This does not mean that Sylvan and Nola's main strategy — solving as many paradoxes as possible on the basis of logical modifications only - cannot be extended with a different kind of switch. Given that what is questionable here are the unqualified applications of Simplification, what is needed to solve this paradox is the adoption of a system

structure of a scientific theory conceived as a set of sentences closed under a relation of logical consequence and the intuitions behind HD-confirmation.

My suggestion is that strengthening the bind between an hypothesis and the background information yields an image closer to what we want to say happens when a piece of evidence HD-confirms some hypothesis. None of it is incompatible with the standard position regarding the structure of theories. There is no reason why we should not have all of $H \circ k, \mathrm{H}$ and $\mathrm{k}$ among the sentences of the general theory. Then, as stated by the reviewer, by Reflexivity, one can get all of them as consequences without issue.

${ }^{11}$ Although see [23] for another account which reconstructs grue as an instance of the tacking paradox.

Australasian Journal of Logic (15:2) 2018 Article no. 3.10 
which restricts Simplification. As a result, the logical switch that the solution of this paradox requires is not relevant, but connexive. This is because one of the characteristic traits of connexive logics is that they reject the validity of Simplification for extensional conjunctions.

Thus, when one makes the switch, then one is in a position to argue for the dissolution of the paradox, as one need only explain that unrestricted Simplification is what allowed the suspicious step from $h \wedge j \Vdash e$ and $h \wedge j \Vdash j$ to $h \wedge j \Vdash e$ and, consequently, to the corresponding confirmation relations. One issue that arises here is that the rejection of Simplification is found to be too radical, even by relevantist lights. Connexivists often try to soften the blow by stating that they do believe there are legitimate applications of Simplification, namely, those in which the conjuncts are relevant to each other. Relevant Logics and their Rivals [33] offers a discussion of the ways in which those who endorse (traditional) connexivism have tried to recover at least some applications of Simplification, with overwhelmingly negative results. ${ }^{12}$

Regardless, there is one key insight to be recovered from this dive into the possibility of a connexive logic underlying a theory of confirmation: a sophisticated version of connexivism called interaction connexivism complements what (traditional) relevant logics do for the Raven and Grue paradoxes. What makes interaction connexivism special is that it focuses on suppression rules rather than on augmentation ones. While it is true that the connexive rejection of Simplification captures only one of many possible senses in which conjunctions might be understood, the claim is not that this is the only legitimate understanding of conjunctions. The downside of this insight is that, as we will see later, this might be more logical revisionism than any confirmation theorist is ready to accept.

\subsection{Some issues}

So far, we have seen that one of the most important questions that have been guiding the quest for a truly comprehensive theory confirmation has been the solution to the paradoxes of confirmation introduced in the first section. We also saw that the traditional discussion, which presupposes that the intuitive conditions on a theory of confirmation - (CL) included - are beyond any doubt, almost unanimously points towards BCTs as our best option for a comprehensive theory of confirmation. ${ }^{13}$ Opposite to the traditional discussion, we found that Sylvan and Nola's counterproposal was that the rejection of (CL) could yield a superior non-Bayesian theory of confirmation in that it could provide a uniform solution for all paradoxes of confirmation.

However, we also found a wrinkle in the "relevant switch" strategy, and an

\footnotetext{
${ }^{12}$ Under a very specific view of connexivism and negation ([20]), failures of Simplification arise from contradictions. Here, the suggestion of an anonymous referee of using a consistency operator might be of help. The problem with the suggestion is that not every failure of Simplification involves explicit contradictions, or even inconsistent information. For more on the discussion of the failures of Simplification, see [17, 42, 44].

${ }^{13}$ Though see for some doubts regarding this point.
} 
important one. In the end, Sylvan and Nola's solution is indeed uniform if one considers two factors:

1. At no point does it require anything over and above replacing classical logic with either a relevant or a connexive one.

2. Following Sylvan's own classification of relevant logics, both the relevant and the connexive switch required for the solution of all three paradoxes count as switching to a (broadly) relevant logic.

This is because Sylvan considers the class of (broadly) relevant and (broadly) connexive logics as one and the same, as the relevance requirement can be fulfilled though the connexion requirement and vice versa. What narrowly distinguishes relevant from connexive logics are the schemas that each reject. This means that narrowly relevant logics "[...] are at bottom connexive logics which solve the paradoxes through the (criticism and) rejection of the principles of Disjunctive Syllogism, Antilogism, and the like" [29, p. 394]. ${ }^{14}$ On the other hand, narrowly connexive logics accomplish the same goal by either rejecting or qualifying Simplification and (typically) Addition. ${ }^{15}$

But, for all of the solution's uniformity, focusing on that aspect makes us overlook another more worrying one: it is very difficult to develop a case for the rejection of Simplification that goes beyond the paradox that is solved with its rejection. The most convincing independent counterexample that connexive logic can offer against Simplification is tied to very non-classical views of negation and conjunction, namely, the cancellation view of negation [31, 33] and the holist view of conjunctions [cf. 33, p. 82]. The cancellation view of negation states that $\neg P$ cancels whatever content $P$ has. ${ }^{16}$ On the other hand, the connexive view of conjunctions sees them as having less content than that of the both conjuncts taken together, as opposed to the holist view, which states that conjunctions have at least as much content as their conjuncts do [cf. 33, pp. 92-94]. It is not straightforward that any of these views of the connectives are at play in contexts of confirmation. At the very least, specific real life examples that involve any of this views are needed so that the case for them gains strength.

Even if a case for these non-classical views of negation and conjunction could be developed, the proposal faces another perhaps more pressing difficulty. Consider that the broad uniformity of Sylvan and Nola's paradox-free confirmation theory depends on two logical switches instead of one. This is important, because insofar as connexive logics are the ones which readily reject Simplification,

\footnotetext{
${ }^{14}$ There is a significant amount of literature available on the relevant rejection of Disjunctive Syllogism (for example, [24] and [30]). In comparison, the availability of literature on the rejection of Simplification and Addition is scarce, revealing that the proposal is neither wellstudied nor understood.

${ }^{15}$ One can take the classification further, as there are also modern connexive logics, which are those logics characterized by the fact that they validate theses such as Aristotle's and Boethius' independently of their rejection (or inclusion) of Simplification and its dual.

${ }^{16}$ Contrast with the classical complement view of negation, which states that $\neg P$ contains everything but $P$.
} 
they are the only ones that can provide a solution for the irrelevant conjuncts paradox. Conversely, insofar as only narrowly relevant logics reject Antilogism for extensional conjunctions from the outset, they are the only ones able to solve both the Raven paradox and extensional grue. ${ }^{17}$ Elsewhere, remarks made by Sylvan (and others) are compatible with his acceptance of a variety of connectives that embody the different senses in which we typically use logical vocabulary within natural language.

Regardless, the same cannot be said of those who endorse the original conditions for a theory of confirmation. Those who think (CL) is an adequate requirement for our best theory of confirmation to uphold are not only endorsing classical logic, but often also believe that classical logic is the 'One True Logic'. It is one thing to say that what is needed to have a paradox-free confirmation theory amounts to one logical switch, say, from classical logic to FDE. But it is quite another to say that what is actually needed is a series of logical switches that fall under the umbrella of relevance preservation. This issue is not brought up as a courtesy to the classicalists, as it is in general important to notice that at this point, the discussion has shifted from the simple rejection of (CL) - in other words, from the rejection of the adequacy of classical logic - to the rejection of (CL) conjoined with the rejection of the assumption that there is a single logical system which will offer us the tools we need to develop a decent theory (at least when restricting ourselves to the context of confirmation).

This shift is important because it no longer hinges on discussions within the context of confirmation theory. Instead, it shows that, as long as an important part of our attempts to provide a comprehensive theory of confirmation stem from our intent to provide some kind of logical analysis of the notion - by relating it, for example, with our notion of logical entailment - then our stance on matters such as the adequacy of classical logic and whether there is a logic that can be said to be the 'One True Logic' will have an import on what we have to say about a the proper analysis of confirmation. These discussions have been extensively written about, so they will be not revisited here. Moreover, no particular stance will be extensively defended over and above what Sylvan and Nola have to say on the matter. That is, the focus will be on the consequences of assuming not only that classical logic is inadequate as the base of a satisfactory confirmation theory, but also that no single logic might be up to the task (call this, 'the radical stance'). With this assumptions in mind, in what follows, the status of BCTs will be reexamined from both the traditional and the radical stance on the debate.

\section{Should we give up BCTs altogether?}

My contention is that the short answer to the question in the title of this section from both sides of the debate should be "no". This is because, at least,

\footnotetext{
${ }^{17}$ I'll assume that, if every instance of definitional grue, blite and emerose involves Addition as well as Disjunctive Syllogism, then both kinds of logics are equally capable of blocking those derivations.
}

Australasian Journal of Logic (15:2) 2018 Article no. 3.10 
the core idea of distributions of probability as degrees of certainty is worth preserving; and this is so regardless what one makes of (CL). Those coming from the traditional side of the discussion, should not find this surprising, as it was already seen that classicalists readily admit that there is something to be said for BCTs (or at least classical probabilistic theories of confirmation) as providing the most sophisticated analysis of confirmation which satisfies most of the conditions imposed on a comprehensive theory. ${ }^{18}$ The novelty should come from the opposite side, as this seemingly started as a dispute for the title of the theory best able to handle the paradoxes of confirmation, and, when compared with Sylvan and Nola's proposal in this aspect alone, no classical probabilistic theory should stand a chance.

We should remind ourselves, however, that a comprehensive theory of confirmation should do more than offer a solution to the paradoxes. We have already mentioned that, apart from offering a truly flexible and sophisticated framework for modelling confirmation relations, BCTs have had such a widespread adoption because of two main reasons:

1. Confirmation as probability has a strong appeal because an important aspect of our intuitive notion of confirmation is that it can come in degrees.

2. Out of all the proposals that satisfy every condition imposed, BCTs are some of the best behaved in the presence of paradox.

Taking this into consideration, my proposal is as follows: those revisionists willing to reject (CL) have no reason for rejecting even the core of BCTs, namely, the idea that an important part of our intuitive notion of confirmation is that it should be quantifiable, as it rarely is an all or nothing matter. Likewise, they should take into consideration that the fine-grained analysis provided by a Bayesian frameworks preserves some other desirable conditions, such as (CCC). Revisionists can, however, contest the idea that BCTs are worth endorsing because they can behave better than their rivals when it comes to the paradoxes of confirmation. For starters, one can argue 'behaving better than x' is far from being equivalent to 'providing a solution to the paradoxes', let alone providing an unified solution to them.

But there is more to this criticism, as even if one concedes that a good explanation is sometimes as good as an uniform solution, when one dives into the details of the Bayesian account, one finds that the improvement over instantial confirmation and HD-confirmation is not really significant. In what follows, the standard Bayesian responses to the three paradoxes we have considered along the way will be reviewed. Along the way we will find that even when an explanation meant to soften the blow of the paradox is provided, the core issue diagnosed by Sylvan and Nola remains. This will be taken to mean two things. First, that BCTs can benefit from the relevant and connexive switches as much as any other confirmation theory can, under the assumption that solving the paradoxes is desirable and that the only actual strategy that can provide such a solution

\footnotetext{
${ }^{18}$ Sprenger [38] introduces the other side of the discussion. His aim is to show the incompleteness of a confirmation theory that encompasses Bayesian but not qualitative methods.
} 
is a logical revision. Also, that BCTs, as any other confirmation theory looking to uphold (CL), can be accommodated within the broader debate concerning logical revisionism.

\subsection{Third time's a charm: the paradoxes revisited}

While BCTs can claim that they handle the Raven Paradox better than their qualitative ancestors,${ }^{19}$ they still accept some weaker version of (PAR), namely, that both a black raven and a pink flamingo offer some - albeit not the samedegree of confirmation for "All ravens are black". This 'standard' response is due mainly to Hosiasson-Lindenbaum (but see also [40] for a discussion of HosiassonLindenbaum's insight), and says that, while it is true that white sneakers confirm a hypothesis about black ravens, they do so by such a small amount, that it is actually negligible. ${ }^{20}$

So far, so good; as this explanation closely (though not exactly) matches our intuition that we should focus on ravens and their color - rather than on sneakers - when looking for evidence that supports our generalization. It is also a familiar strategy, for here, as in the case of HD-confirmation, the burden is on the background information and auxiliary hypotheses, as they are the ones that determine the probabilities assigned to reports of black ravens and non-black objects, which in turn determine how much confirmation each piece of evidence is able to offer.

However, the point can be pressed on two counts. The first, based entirely on our intuitions, is that while it seems legitimate to consider that while this response to the paradox is adequate, one could always demand a solution that answers to the stronger intuition, that the positive instances that confirm "All non-black things are non-ravens" are irrelevant to the confirmation of "All ravens are black", preferably without resorting to issues concerning the naturalness or degree of entrenchment. Alas, Bayesians of all stripes may simply reply that this is as good as BCTs get, so that one should be grateful that the theory does not depart too far from our intuitions on this one.

However, this makes most BCTs susceptible to the following comeback, based on the following argument due to [15]:

1. By the canonical responses, the sighting of a black raven confirms "All ravens are black" to a greater degree than a non-black non-raven (say, a white sneaker), does.

2. As is widely known, by Antilogism (or Contraposition), "All ravens are black" is logically equivalent to "Every non-black object is a non-raven".

\footnotetext{
${ }^{19}$ And that they can, as this is a claim that is sufficiently backed by the vast amount of literature discussing Bayesianism and the paradox. To wit, begin with Vranas' review on the available literature [43].

${ }^{20}$ This intuition has been refined in several places, for instance, [9]. Furthermore, [26] presents an account in which instances of both black non-ravens and non-black non-ravens are neutral, rather than offering any degree of (dis)confirmation whatsoever.
} 
3. Most Bayesians - and confirmation theorists in general - admit some version of $(\mathrm{EQ})$. In particular, their version of $(\mathrm{EQ})$ tends to be formulated as follows: For all $p$ and $q$, if $p$ and $q$ are logically equivalent, then $\operatorname{Pr}(p)=\operatorname{Pr}(q)$.

4. As a result, updating the probabilities of "All ravens are black" and "Every non-black object is a non-raven" (which are the same), using the same piece of evidence and by the same update measure will yield that, without further qualification, the sighting of a black raven confirms "Every nonblack object is a non-raven" to a greater degree than the sighting of a non-black non-raven (say, a white sneaker), does. Or worse, as following Rinard [26] non-black non-ravens turn out to be neutral confirmation-wise.

This argument is not meant to be an insurmountable obstacle for Bayesian theorists. Strevens [40] suggests that Bayesians should remain agnostic about the equivalence between an hypothesis and its contrapositive. I think he is along the right track, as (EQ) gets Bayesians dangerously close to the problem of logical omniscience. Regardless, the bite of this argument lies in the fact that, if logic is not in general revisable and this is a situation in which we have gone from one hypothesis to the other by means of logic alone, Bayesians are hard-pressed to find an explanation that is not ad hoc and, most importantly, that does not fly in the face of their previous commitment to (CL) as shorthand for their commitment to classical logic being the only logic up to the task of adequately underlying a satisfactory theory of confirmation.

My guess is that even Bayesians who openly commit to (EQ) have an attitude that is closer to Strevens' agnosticism in practice. They will recognize the (classical) equivalence in terms of conceding that both a statement and its contrapositive should be assigned the same initial probability, but then they will implicitly attempt to block Laetz' scenario by rejecting that we should keep the same distribution of probabilities when considering the contrapositive, as it is quite unacceptable to think that a black raven offers a higher degree of confirmation to "All non-black things are non-ravens" than a white shoe does.

If this account is along the right lines, then Sylvan and Nola's proposed move is not as radical as it might initially seem. Laetz' argument only serves to make explicit that Bayesians of all stripes have already noticed that even with all sorts of restrictions for $(\mathrm{NC})$ in place, $(\mathrm{EQ})$ - a desirable principle from a structural viewpoint - keeps on bringing trouble about. Sylvan and Nola are pushing qualitative confirmation-theorists to resist Bayesianism by switching to a conditional which does not admit equivalence with Contraposition in order to avoid the original paradoxical conclusion and thus be paradox free.

On to the irrelevant conjuncts paradox (for background discussion, see [5, 6]). Here, the focus shifts from the conditional to (the classical view of) conjunctions, and more precisely, to the availability of the pesky Simplification Rule. Bayesians share the woes of HD-confirmation theories in that, in order to preserve the notion that induction is the converse of (classical) deduction, they allow the consequence relation to be monotonic. This has the unpleasant result 
that, in both cases, Augmentation allows the inclusion of irrelevant premisses, which are consequently said to be confirmed by any given conclusion that happens to be in the right side of the implication. The key condition here is (SCC) which is often considered as a truism, one that enables us to say that by Simplification, conjuncts are logical consequences of any conjunction and are thus individually confirmed by anything that confirms the conjunction.

Evidently, before starting an assault on (CL), it is also reasonable to question the status of (SCC). However, this is quite problematic, as there does not seem to be a straightforward way to restrict the condition. For how are we to choose which of the consequences of a theory are confirmed and which are not? As an attempt to at least soften the impact that irrelevant conjuncts have on BCTs, Strevens [40] essays some possible escapes available to the Bayesian theorist. The first one is to assume that one is simply able to make the calculations to determine how much support (if any) is received by each conjunct. The inadequacy of this alternative stems from the fact that, even if making the calculations is a straightforward approach to solving the problem, it seems legitimate to ask for a strategy that is not performed on a case-to-case basis.

The second and third alternatives concern determining what it means for a conjunct to be relevant. First, one can try to capture irrelevance using the notion of 'probabilistic independence'. This results in an explanation that is not really insightful, for as Strevens notes, under this assumption, for $\mathrm{j}$ to be irrelevant to $\mathrm{h}$ (and e), is just for $\mathrm{j}$ not to be confirmed by e. The last attempt is the most fruitful of the three: what we are trying to capture is that if $\mathrm{j}$ is irrelevant to $\mathrm{h}$, then the es that confirm $\mathrm{h}$ cannot also confirm $\mathrm{j}$. This is because it seems reasonable to think that $\mathrm{j}$ is irrelevant to $\mathrm{h}$ if $\mathrm{e}$ is irrelevant to $\mathrm{j}$ (where e confirms h).

No matter how one tries to reconstruct the issue, the parallels with Sylvan and Nola's arise without too much effort. One can see their proposal as simply stating that ( $\mathrm{SCC}$ ) is along the right lines, namely, that it is reasonable to expect that a given piece of evidence confirms all the logical consequences of the hypotheses it already confirms. What we should be aiming to do, Sylvan and Nola would say, is to restrict our logical vocabulary in such a way that we are only ridding ourselves of (CL) - something that we should be doing anyway. Notice that, as the culprit here is Simplification, the discussion will unavoidably lead us back to the uncharted territory of connexive logics. This, while quite esoteric, is not necessarily a bad thing, as there are a couple interesting parallels to be drawn.

The first one concerns the traditional connexivist reasons to restrict Simplification. In a nutshell, the concern of connexivism is with the loss of sufficiency that the combination of augmentation and supression principles brings about. Translated into the context of confirmation relations, the connexivist story makes perfect sense as the trouble is that, once a relation of the form $H \wedge k \Vdash e$ obtains, we are almost certainly in the face of necessary and sufficient conditions for e. The introduction of irrelevant information may be harmless until one realizes that, conjoined with supression principles such as Simplification, it potentially has the loss of sufficiency (i.e. relevance, in some sense) as 
a consequence.

The other parallel has to do with the connexive treatment of conjunction. The rejection of Simplification has its roots not only in providing a solution to the loss of sufficiency, but also in a particular view of conjunctions. Classicalists typically endorse a holist view of conjunctions, in which the conjunction, being essentially a set theoretic union, has at least as much content as both of its conjuncts together do. In contrast, connexivism treats conjunctions as having less content than the union of its conjuncts [cf. 33, p. 82]. This has to do with the fact that connexivism usually endorses interactionism regarding the connectives, and the fact that connexivism often adopts a cancellation view of negation. ${ }^{21}$

For example, when probabilistic calculi are interpreted as being about events, the parallel becomes clear. The joint probability of two independent events, one of which has a probability of $1 / 2$ and another which has a probability of $1 / 5$ is $1 / 10$. If we interpret the first probability as "containing" the 5 events out of 10 in which a happens, and the second as "containing" the two times that b happens, we will get to the conclusion that the joint probability only contains a single event out of the ten originally considered, namely, the one in which both $\mathrm{a}$ and $\mathrm{b}$ happen. Unfortunately, for all their similarities, the difficulties with the adoption of a connexive logic discussed above transfer to this discussion untouched.

Finally, we need to examine what happens with grue and its kin (See [35, 39] for some background). Again, BCTs find themselves in a situation in which they are unable to give us a general result which lets us discern between 'green' and 'grue'. At most, Bayesians can tell us that if we are previously predisposed to projecting predicates such as 'green' into the future, then updating will preserve our initial bias, no matter what (cf. Strevens $[39,40]$ ). This chains the discussion nicely with one of BCTs own problems, one that has nothing to do with paradox: the problem of the priors [cf. 18, pp. 428-32]. While there is nothing intrinsically wrong with expecting the problem of the priors to have a solution and thus for BCTs to be able to provide a complete solution to this kind of paradox, there is also no reason why we should not revisit Sylvan and Nola's proposal.

It is important to notice that not only do we lack reasons for discarding it; actually, Sylvan and Nola's proposal fares better than the traditional Bayesian solution in at least one respect: instead of explaining the problem away, it stops it in its tracks, as the invalidity of Antilogism and Disjunctive Syllogism simply block each and every reasoning associated with grue, blite, and related predicates. This, together with the potential for blocking the reasoning behind the Raven paradox in one fell swoop, should increase the appeal of switching the logic that underlies even Bayesian accounts of confirmation.

\footnotetext{
${ }^{21}$ Briefly, interactionism opposes separatism, namely, the thesis that every connective is such that its behavior is completely independent of the rest [cf. 33, pp. 92-95].
}

Australasian Journal of Logic (15:2) 2018 Article no. 3.10 


\subsection{Final remarks}

But, for all the enthusiasm that has been expressed here in favor of the so-called 'relevant switch', the proposal lacks any noticeable punch if we are unable to offer at least some guidelines as to its implementation. This is especially important when one considers that one of the things that BCTs have going for them is that classical probability, as all classical theories, has been thoroughly studied and is very well understood. Fortunately, the idea of non-classical Bayesian confirmation is not new. One can already find proposals to reject (CL) in $[16,21,27]$ and [3]. These proposals are mainly motivated by the claim that epistemic agents should be able to accommodate inconsistent beliefs. However, some of them, like [16] and [27] could provide an adequate starting point, as the models they present are based on FDE, a paraconsistent logic that serves as the implication-free fragment of many relevant logics.

If anything, what has been said along this section should function as further motivation for Mares' project, as both his aim and Sylvan and Nola's are actually complementary. It should also be noted that this goes beyond the mere convenience of having a probabilistic theory of confirmation which is able to block nearly all paradoxes of confirmation and model paraconsistent confirmation relations at the same time. The existence of both proposals actually means that one can contest (CL), and, more precisely, the adequacy of classical logic as the logic that underlies a comprehensive theory of confirmation, on two counts.

The first one is, as Mares already stated, that it is unrealistic to expect that any given epistemic agent is able to remain consistent without exception. Real life examples show us that paraconsistent confirmation relations do happen, so it is desirable that our preferred theory of confirmation does not exclude them from the outset, just because there is an apparent need to preserve classicality. The other one is, as has been developed, that paradoxes are an unwelcome consequence of theories that strive to preserve classicality, which commits us to suspicious principles that we are burdened with for no other reason than our choice of logic.

Closer inspection reveals that these are particular theses that stem from more general arguments that belong to the general debate on the adequacy of classical logic as the basis for philosophical and even scientific theories, the relationship of logic and reasoning and on logical pluralism. Mares' proposal is concrete evidence that the need for paraconsistency arises in the context of confirmation, just as it has previously arisen in other particular discussions which belong to areas such as the philosophy of science and the philosophy of mathematics. Likewise, the usage of a relevant logic in order to dissolve paradoxes that arise in the context of confirmation is a concrete example of the usefulness of nonclassical logics in diverse areas of philosophy. Sylvan himself advocated such a project, both in his work with Nola and elsewhere.

Ideally, within a probabilistic setting, one should be able to extend the argument to match the qualitative version. When discussing the consequences of Sylvan and Nola's project for qualitative confirmation, we found that a potential drawback (for classicalists and logical monists alike) was that the actual 
wholesale solution to the problems of confirmation required not one, but two independent - albeit broadly relevant - logical switches. It was seen that rejecting one of the switches almost certainly meant losing the solution to one of the three paradoxes, as it is unclear that a connexive logic alone can invalidate Antilogism, and no narrowly relevant logic rejects, or even restricts, Simplification.

As matters stand, we find ourselves in an analog situation. Taking Mares' probabilistic models as a starting point means switching from classical logic to FDE, a narrowly relevant logic. This leaves the problems with Simplification untouched, as FDE does not do away with the rule. Sylvan and Nola warn us that connexive confirmation is uncharted territory, and they do so with good reason. Sylvan is definitely amenable to the idea that both relevant and connexive logical connectives recover different aspects of our actual usage of logical vocabulary, and as such can be said to complement each other. However, he is also no stranger to the difficulties of even providing the connexive connectives with a satisfactory interpretation [See 28].

It looks like his own remarks on the suspiciousness of Contraposition turn on him. Just as he said that, not considering the existence of countermodels to Contraposition, the case for the rejection of the rule was as strong as whatever real-life counterexamples could be developed against it. In an analogous manner, one could say that, the existence of semantics notwithstanding, the case for the connexive connectives is only as strong as whatever philosophical interpretation can be offered for them.

This is because, we are here dealing with another argument involving a bridge principle. No one doubts that classical logic (as a formal language) is an adequate home to the semantic and naïve set theory paradoxes. The paradoxes of confirmation, however, arise in natural language (hence, the availability of Fitelson's main argument in [8]). Consequently, while there might be all sorts of formal languages available in which the confirmational paradoxes cause no trouble, we still need the extra step that convincingly connects what happens there with what happens in our very own natural language. Otherwise, it looks like we have done nothing more than find a (set of) formal language(s) completely unrelated to our own which can avoid a problem ours cannot get rid of. ${ }^{22}$

Given that not much changes when going from the qualitative to the quantitative context, the present discussion offers no additional motivations for the connexive switch. Even if an adequate interpretation could be provided, a couple of problems (analog to those of the qualitative discussion) remain. The first one is that we don't have a thorough understanding of exactly what makes a given connective a conjunction, nor of how is it that multiple connectives of the same kind interact within a given context. This lack of understanding turns the probabilistic variant of the problem into something we understandably would not like to get into, at least not until we have the truth-functional case figured out.

\footnotetext{
${ }^{22}$ This resonates as a variant of an argument frequently thrown at the ternary relation semantics. For more on that particular discussion, see [2].
}

Australasian Journal of Logic (15:2) 2018 Article no. 3.10 
The second one is that the very acceptance of contexts in which different connectives interact is, again, part of a broader discussion on the nature of the logical connectives, and of logic itself. At their core, BCTs imply both classicalist and monist commitments, and Mares' probabilistic models imply the loss of classicality but not of monism. So long as the opponents in the discussion are classicalists and monists, and we struggle to provide a reasonable interpretation for some of the elements that we have introduced, it will be very difficult to build a strong case for a project like the one Sylvan and Nola propose, truth-functional or not.

Thus, we find ourselves revisiting another discussion that was already touched upon, perhaps with the addition of the following morale: the choice between truth-functionality and probability is largely independent from one's stance on logical pluralism. And more generally, the features that we recognize as desirable in a notion of confirmation should also be largely independent of our logical commitments. This is not to say that we are thus denying that confirmation has any relationship with logic. It just means that we should recognize that we seldom stop to examine the bulk of the commitments we are making with the adoption of a given theory. At most, we tend to examine only those that are relevant for our most immediate purposes.

This summarizes the unsurprising popularity of BCTs quite neatly: when in the market for a theory of confirmation, we pay attention to the flexibility of the framework and to its usefulness, and the fact that the logic up to the task of providing the required theory is classical hardly is simply taken for granted. The attitude is not bad per se, for there are compelling reasons to trust on the power of classical logic. It might even turn out that the theories based on classical logic are the best alternatives no matter what. However, we cannot take that as a truism, as it amounts to a philosophical thesis like any other. This means, that, as happens with every other philosophical thesis, we are not entitled to its assertion until we have made sure that we did not leave any (logical) stone unturned.

\section{Conclusions}

Sylvan and Nola suggest that we should adopt a non-classical solution to the confirmation paradoxes as an alternative to BCTs. Here I have argued that while their paradox-solving strategy, replacing classical logic with a relevant (or connexive) one, is along the right lines, it should by no means be regarded as a replacement for quantitative confirmation theories. Additionally, I have suggested that neither Sylvan and Nola's proposal nor mine should be taken as a definite answer to the issue. This is so especially in light of Fitelson's argument (and competing account of BCT) and a similar one relating non-classical formal languages and natural languages.

In Sections 2 and 3, some preliminaries on confirmation theory and the paradoxes were developed. In Sect. 2 the focus was on highlighting the core of each of the available strategies for providing a theory of confirmation. One 
of the main purposes of this section was to show, that even if the matter is often construed as that of a theory replacing the ones that came before it, some have pointed out that they respond to different issues and thus might be worth preserving. However, this section also recognizes that, when push comes to shove, no theory provides a more powerful framework than BCTs. The following section introduces the basics on the paradoxes of confirmation. While this might seem unnecessary, it at least ensures uniformity in the discussion.

Following the traditional discussion on confirmation and its paradoxes, Sect. 4 introduced our two accounts of paradox resolution. First, the canonical solutions for the Raven and Grue paradoxes were reconstructed. Then, Sylvan and Nola's work was revisited. The caveat was that their dismissal of BCTs is done not by exposing a flaw in the theories, but by challenging the unquestioned adoption of (CL). As a result we took Sylvan and Nola to be arguing for something which is in many aspects compatible with the core intuitions of Bayesianism, so it does not give convincing grounds for its rejection.

A downside that came up during the reconstruction of Sylvan and Nola's proposal that it turned out to be more revisionist than it originally appeared. I found this to be a negative consequence of the proposal for some reasons: (1) the difficulties associated with the adoption of a connexive logic; (2) where the opponents are classicalists and logical monists, such a revisionist proposal needs to be strengthened with compelling motivations for its adoption; (3) a positive case for the proposal is non-existent, partly because there is still work to done and partly because of the obstacles it needs to overcome. The upshot was that, even if a single relevant switch is taken up, a confirmation theory (of any kind) based on a relevant logic fares much better in paradox resolution than its classical rivals.

Finally, Sect. 5 aims to change the direction of the discussion. While it is true that qualitative theories based on relevant logics fare better in the face of paradox, there are independent reasons to insist on the core of BCTs as an appealing feature of our best theories of confirmation. However, it can be shown that even after all their paradox-handling, some counterintuitive intuitions remain. Most importantly, there is no current canonical solution to the irrelevant conjuncts paradox. Once again, all of this issues can be solved combining the Bayesian and the non-classical strategies. Unfortunately, this is easier said than done, so the section closes with some remarks regarding non-classical BCTs, the other Harman-style argument, and the relationship between this discussion and logical pluralism. ${ }^{23}$

\footnotetext{
${ }^{23}$ I have been endorsing logical pluralism throughout, mainly because it is the position I'm sympathetic with. There is no doubt that one can remain a logical monist, but my guess is that a single logic that attempts to solve all paradoxes of confirmation in one fell swoop will likely be too weak to be useful or even appealing for most. Furthermore, it is quite likely that the resulting logic will be too weak for someone to defends that classical logic is mostly right about other aspects of scientific enquiry (like mathematics).
} 


\section{References}

[1] W. A. Bass. Relevant to Grue? International Logic Review, 18(35), 1987.

[2] Jc Beall, Ross Brady, J. Michael Dunn, A. P. Hazen, Edwin Mares, Robert K. Meyer, Graham Priest, Greg Restall, David Ripley, John Slaney, and Richard Sylvan. On the Ternary Relation and Conditionality. Journal of Philosophical Logic, 41(3):595-612, June 2012.

[3] Juliana Bueno-Soler and Walter Carnielli. Paraconsistent probabilities: consistency, contradictions and Bayes' theorem. Entropy, 18(9):325, 2016.

[4] Vincenzo Crupi. Confirmation. In Edward N. Zalta, editor, The Stanford Encyclopedia of Philosophy. Metaphysics Research Lab, Stanford University, winter 2016 edition, 2016.

[5] Vincenzo Crupi and Katya Tentori. Irrelevant Conjunction: Statement and Solution of a New Paradox*. Philosophy of Science, 77(1):1-13, 2010.

[6] Branden Fitelson. Putting the Irrelevance Back Into the Problem of Irrelevant Conjunction. Philosophy of Science, 69(4):611-622, 2002.

[7] Branden Fitelson. The Paradox of Confirmation. Philosophy Compass, 1 (1):95-113, January 2006.

[8] Branden Fitelson. Goodman's "New Riddle". Journal of Philosophical Logic, 37(6):613-643, 2008.

[9] Branden Fitelson and James Hawthorne. The wason task (s) and the paradox of confirmation. Philosophical Perspectives, 24(1):207-241, 2010.

[10] I. J. Good. The white shoe is a red herring. The British Journal for the Philosophy of Science, 17(4):322-322, 1967.

[11] I. J. Good. The white shoe qua herring is pink. The British Journal for the Philosophy of Science, 19(2):156-157, 1968. ISSN 00070882, 14643537.

[12] Carl G. Hempel. Studies in the Logic of Confirmation (II.). Mind, 54(214): 97-121, 1945.

[13] Carl G. Hempel. Studies in the Logic of Confirmation (I.). Mind, 54(213): 1-26, 1945.

[14] Janina Hosiasson-Lindenbaum. On Confirmation. The Journal of Symbolic Logic, 5(4):133-148, 1940.

[15] Brian Laetz. Does the Bayesian solution to the paradox of confirmation really support Bayesianism? European Journal for Philosophy of Science, 1(1):39-46, January 2011.

[16] Edwin D. Mares. Paraconsistent probability theory and paraconsistent Bayesianism. Logique et Analyse, 40(160):375-384, 1997. 
[17] Everett J. Nelson. Intensional relations. Mind, 39(156):440-453, 1930. ISSN 00264423, 14602113.

[18] John Norton. Challenges to Bayesian Confirmation Theory. In Prasanta S. Bandyopadhyay and Malcolm R. Forster, editors, Handbook of the Philosophy of Science, Vol. 7: Philosophy of Statistics, pages 391-440. Elsevier B.V., 2011.

[19] Samir Okasha. Experiment, observation and the confirmation of laws. Analysis, 71(2):222-232, 2011.

[20] Graham Priest. Negation as Cancellation, and Connexive Logic. Topoi, 18 (2):141-148, September 1999.

[21] Graham Priest. In Contradiction: A Study of the Transconsistent. Oxford University Press, 2006.

[22] Graham Priest. Logical Pluralism Hollandaise. The Australasian Journal of Logic, 6, December 2008.

[23] Murali Ramachandran. A neglected response to the paradoxes of confirmation. South African Journal of Philosophy, 36(2):179-185, June 2017.

[24] Stephen Read. What Is Wrong with Disjunctive Syllogism? Analysis, 41 (2):66-70, 1981.

[25] Stephen Read. Relevant Logic: A Philosophical Examination of Inference. Blackwell, 1988.

[26] Susanna Rinard. A New Bayesian Solution to the Paradox of the Ravens. Philosophy of Science, 81(1):81-100, January 2014.

[27] Lesley Roberts. Maybe, maybe not: Probabilisitc semantics for two paraconsistent logics. In D. Batens, C. Mortensen, G. Priest, and J. P. Van Bendegem, editors, Frontiers of Paraconsistent Logic. Research Studies Press, 2000 .

[28] R. Routley and H. Montgomery. On Systems Containing Aristotle's Thesis. The Journal of Symbolic Logic, 33(1):82-96, 1968.

[29] Richard Routley. Semantics for connexive logics. I. Studia Logica, 37(4): 393-412, December 1978.

[30] Richard Routley. How Disjunctive Syllogism Can Be Seen as a Fallacy of Relevance. Bulletin of the Section of Logic, 10(4):144-149, 1981.

[31] Richard Routley and Valerie Routley. Negation and contradiction. Revista colombiana de matematicas, 19(1-2):201-230, 1985.

[32] Richard Routley and Richard Sylvan. Exploring Meinong's jungle and beyond: an investigation of noneism and the theory of items. Australian National University, 1980. 
[33] Richard Routley, Val Plumwood, Robert K. Meyer, and Ross T. Brady. Relevant logics and their rivals. 1982.

[34] Gerhard Schurz. Philosophy of Science: A Unified Approach. Routledge, 2013.

[35] Elliott Sober. No model, no inference: A Bayesian primer on the grue problem. Grue, pages 225-240, 1994.

[36] Jan Sprenger. Hempel and the Paradoxes of Confirmation. In Handbook of the History of Logic, volume 10, pages 235-263. Elsevier, 2011.

[37] Jan Sprenger. Hypothetico-Deductive Confirmation. Philosophy Compass, 6(7):497-508, 2011.

[38] Jan Sprenger. A Synthesis of Hempelian and Hypothetico-Deductive Confirmation. Erkenntnis (1975-), 78(4):727-738, 2013.

[39] Michael Strevens. Bayesian confirmation theory: Inductive logic, or mere inductive framework? Synthese, 141(3):365-379, 2004.

[40] Michael Strevens. Notes on Bayesian Confirmation Theory. June 2017.

[41] Richard Sylvan and Robert Nola. Confirmation without paradoxes. Advances in Scientific Philosophy, Rodophi, Amsterdam, pages 5-44, 1991.

[42] Bruce ER Thompson. Why is conjunctive simplification invalid? Notre Dame Journal of Formal Logic, 32(2):248-254, 1991.

[43] Peter BM Vranas. Hempel's raven paradox: A lacuna in the standard Bayesian solution. The British journal for the philosophy of science, 55(3): 545-560, 2004.

[44] Heinrich Wansing, Hitoshi Omori, and Thomas Macaulay Ferguson. The Tenacity of Connexive Logic: Preface to the Special Issue. Journal of Logics, pages 279-296, 2016.

[45] J. Robert G. Williams. Probability and Nonclassical Logic. In Alan Hajek and Christopher Hitchcock, editors, The Oxford Handbook of Probability and Philosophy. Oxford University Press, Oxford, 1 edition, 2016. 